\title{
Design of smart wetting of building materials as evaporative cooling measure for improving the urban climate during heat waves
}

\author{
Andrea Ferrari ${ }^{1}$, Aytac Kubilay $^{2}$, Dominique Derome ${ }^{3}$, and Jan Carmeliet $^{1, *}$ \\ ${ }^{1}$ Chair of Building Physics, Department of Mechanical and Process Engineering, ETH Zurich, Switzerland \\ ${ }^{2}$ Laboratory of Multiscale Studies in Building Physics, Empa, Switzerland \\ ${ }^{3}$ Department of Civil and Building Engineering, Université de Sherbrooke, Canada
}

\begin{abstract}
An urban microclimate model is used to design a smart wetting protocol for multilayer street pavements in order to maximize the evaporative cooling effect as a mitigation measure for thermal discomfort during heat waves. The microclimate model covers a computational fluid dynamics (CFD) model for solving the turbulent air, heat and moisture flow in the air domain of a street canyon. The CFD model is coupled to a model for heat and moisture transport in porous urban materials, to a radiative exchange model, determining the net solar and longwave radiation on each urban surface and to a wind driven rain model able to determine the wetting flux on each surface during a rain event. We first evaluate the evaporative cooling potential for different pavement systems during normal summer conditions after a long rain event during night in order to select an optimal pavement system. Then, we design a smart wetting protocol answering the questions 'when', 'how much' and 'how long' a pavement should be artificially wetted for having a maximum cooling effect. We found that a daily amount of $5 \mathrm{~mm}$ wetting over 10 minutes in the morning, preferentially between 8:00 and 10:00 am, guarantees a maximal evaporative cooling for one day and night during a heat wave.
\end{abstract}

\section{Introduction}

The cooling demand of buildings is expected to increase dramatically in the future mainly due to further urbanization and climate change. Three main actions may reduce the building energy demand for cooling: (i) the improvement of the building energy performance, (ii) the use of more efficient and/or alternative cooling technologies such as passive and free cooling; (iii) the mitigation of the urban heat island (UHI) effect reducing the outside air temperature especially during extreme heat wave events. UHI refers to higher air temperatures in urban environment compared to rural areas. This increase in temperature is due to an increased sensible heat storage of solar radiation during the day, radiation entrapment in street canyons, less urban cooling due to a decrease in evapotranspiration (less vegetation), a decrease in heat removal by urban ventilation (wind), and a decrease in longwave radiation to the colder sky at night (reduced sky view factor). The UHI effect has an adverse impact on thermal comfort, building energy use for cooling and public health [1]. Mitigating UHI consists in a combination of measures such as the use of high albedo materials, introduction of more evapo-transpirative cooling by vegetation and porous materials and promotion of urban ventilation and shading.

The impact of reflective and permeable building materials on the local urban climate and thermal comfort has been studied by the authors in [2, 3 and 4]. Pedestrian thermal comfort can be evaluated using comfort indices, like the Universal Thermal Climate Index (UTCI). The UTCI represents a human thermal sensation using an equivalent temperature, causing a physiological response of a reference person equivalent to the one in the actual environment [5]. The UTCI depends mainly on mean radiant temperature including solar radiation and longwave radiation from surrounding surfaces on a person and local variables such as air temperature, relative humidity (RH) and wind speed. In these studies, the authors compared the cooling effect by evaporation occurring in different porous urban materials for different wetting conditions.

In dry conditions, the lowest temperature of urban surfaces is obtained for high albedo values of the surface and high thermal diffusivity responsible for a large heat diffusion and storage. In wet conditions, the highest reduction in surface temperature occurs during the first drying phase of a wet material, when liquid transport from inside the material provides sufficient liquid water to the drying surface, so that the material surface remains at high moisture content and at almost $100 \% \mathrm{RH}$. In this period, the drying rate is maximal and depends mainly on the external environmental conditions, such as local air temperature, RH, wind speed and radiation. The needed energy for evaporation during the high drying rate period causes a cooling effect and reduction in surface temperature, called evaporative cooling. Once the liquid within the porous medium cannot anymore replenish the

*orresponding author: cajan@ethz.ch 
material surface, drying enters its second phase. During this second drying phase, the evaporation rate decreases and depends mainly on the vapour permeability of the dry zone of the material. To maximize the evaporative cooling effect, porous materials have to be chosen in such way that the first drying period lasts as long as possible. The potential of evaporative cooling effect due to rain is highest for street surfaces compared to other building surfaces, since they receive not only most rain for evaporative cooling, but also most solar radiation, which enhances the drying process. It is shown in [2] that the street surface temperature can drop by $25^{\circ} \mathrm{C}$ due to evaporative cooling of a brick pavement after being exposed to a rain event of $1 \mathrm{~mm} / \mathrm{h}$ for 10 hours. In turn, the reduction in surface temperature results in a reduction of the air temperature of $2^{\circ} \mathrm{C}$. The reduction in both surface and air temperature leads to an improvement of the thermal comfort. However, evaporation also leads to an increase in RH and decrease in wind velocity, effects, which reduce thermal comfort. A reduction in UTCI comparing dry and wet conditions is found to amount to $3^{\circ} \mathrm{C}$. In [3], the evaporative cooling effect for different porous materials (concrete, brick, soil), rain intensities and durations of wetting are compared. In [4], different multilayer pavement solutions are designed optimizing their moisture transport properties and layering structure in order to guarantee the highest evaporative cooling effect.

In these previous studies wetting by rain is used, which is an uncertain phenomenon during heat waves, since rain events highly depend on weather conditions and cannot be planned. Another option is to artificially wet pavements by stored rain water or use of grey water from buildings. This opens the door for "smart wetting" solutions, where the time of wetting, wetting period and wetting amount are chosen for maximizing the evaporative cooling effect and providing optimal thermal comfort. Artificial pavement watering can be performed e.g. by cleaning trucks $[6,7,8]$ which however may create disturbance of traffic. Therefore, the time of wetting has to be chosen carefully.

In this paper, we study the impact of artificial wetting on the local urban climate in a street canyon during a heat wave period. Artificial watering of the pavement is defined by three parameters: total water amount, which is a result of wetting flux $\boldsymbol{g}_{\text {wet }}$ under a given duration of wetting, time of the day and frequency (number of cycles per day) of application. It is recommendable that the evaporative cooling effect lasts at least for one full day and night cycle so that the wetting frequency can be limited to once a day. In "smart wetting" solutions, the artificial wetting parameters are chosen aiming to maximize the pavement cooling in the first 24 hours while minimizing the amount of water to be used and the duration of watering. For practical reason, such wetting is limited to one watering cycle/day, starting as early as possible and with a short duration to minimize the interference of artificial wetting with urban activities, such as traffic.

The paper is organised as follows. First, the urban microclimate model is described paying special attention to the formulation of adequate boundary conditions at the urban surfaces. Then the case study used in this analysis is described, consisting of the description of the street canyon geometry, the materials used and the climatic conditions. In the results part, we first document the evaporative cooling effect for different pavement solutions, and then focus on the questions of 'how much', 'when' and 'how long' artificial wetting is needed to obtain a maximal evaporative cooling effect during a heat wave for a day and night cycle. Finally, conclusions are formulated.

\section{Model}

In this study, the impact of evaporative cooling of permeable building materials on the local urban microclimate is investigated by means of a fullyintegrated urban microclimate model. The urban domain consists of two subdomains; the air domain and the domain consisting of the porous materials. This porous material domain includes the porous materials building up walls, roofs and ground. The air surrounding the buildings and street forms the air domain. The microclimate model consists in coupling four different sub-models. A computational fluid dynamics (CFD) model solves the turbulent, convective air flow and heat and moisture transport in the air domain. A coupled heat and moisture (HAM) transport model solves the heat and moisture flows in the porous domain. The CFD model uses a steady RANS (Reynolds Averaged Navier Stokes equations) approach, and is coupled to unsteady HAM solutions in the porous domain, where information is exchanged between the models at their interfaces at each calculation time-step. A radiation model determines the net longwave and shortwave radiative heat flux for each surface (urban surfaces and sky) using a radiosity approach and assuming all surfaces to be opaque, grey and diffusive. Multiple reflections of both solar and thermal radiation are considered in order to account for possible radiation entrapment within street canyons. Finally, wind driven rain (WDR) fluxes at the surface of the porous domain are calculated using an Eulerian multiphase model [9], in which the rain phase is considered as a continuum consisting of different rain phases for each raindrop size range, and where each rain phase interacts with wind flow. The four sub-models (CFD, HAM, radiation and WDR) are implemented in OpenFOAM 6.0 [10], where the conservation equations for both air and porous domains are discretized by the finite volume method. A detailed description of this model and its validation can be found in [2, 3 and 4].

\subsection{Boundary conditions at porous surfaces}

The air and porous domains are coupled at their boundaries, called interfaces, where adequate boundary conditions are applied to ensure continuity of heat and moisture fluxes between the domains. The heat flux from the air domain to the interface is given by the sum of the convective heat flux $\boldsymbol{q}_{c o n v}$, the net long- and short-wave radiation fluxes $\boldsymbol{q}_{L W}$ and $\boldsymbol{q}_{S W}$, respectively, the sensible 
heat transfer due to wetting of the surface $\boldsymbol{q}_{\text {wet }}$ and the sensible and latent heat transfer due to vapour exchange $\boldsymbol{q}_{\text {vap. }}$. The heat flux from porous domain to interface consists in conductive and advective heat fluxes $\boldsymbol{q}_{c}$ and $\boldsymbol{q}_{a}$, respectively. The continuity of heat flux at the interface is given by:

$$
\boldsymbol{q}_{c o n v}+\boldsymbol{q}_{L W}+\boldsymbol{q}_{S W}+\boldsymbol{q}_{\text {wet }}+\boldsymbol{q}_{v a p}=\boldsymbol{q}_{c}+\boldsymbol{q}_{a}
$$

The moisture flux from the air domain to the interface is composed of the convective vapour flux $\boldsymbol{g}_{\text {conv }}$ and the water mass flux $\boldsymbol{g}_{\text {wet }}$ due to rain or artificial wetting. The moisture flux from porous domain to interface is given by the sum of liquid and vapour fluxes $\boldsymbol{g}_{l}$ and $\boldsymbol{g}_{v}$. Continuity of moisture flux at the interface leads to:

$$
\boldsymbol{g}_{c o n v}+\boldsymbol{g}_{\text {wet }}=\boldsymbol{g}_{l}+\boldsymbol{g}_{v}
$$

The convective heat and moisture fluxes from the air domain to the interface are obtained from the CFD calculation. The sensible heat flux due to wetting and the sensible and latent heat fluxes due to vapour exchange are respectively obtained by:

$$
\begin{gathered}
\boldsymbol{q}_{\text {wet }}=c_{p l} T_{\text {wet }} \boldsymbol{g}_{\text {wet }} \\
\boldsymbol{q}_{v a p}=\left(c_{p v} T_{s}+L_{v}\right) \boldsymbol{g}_{\text {conv }}
\end{gathered}
$$

where $c_{p v}$ and $c_{p l}$ are the specific heat of water vapour and liquid water, respectively, $L_{v}$ the heat of evaporation, $T_{w e t}$ the water temperature during wetting and $T_{s}$ the surface temperature. Continuity of heat and moisture fluxes between the models is guaranteed by sequentially solving adequate boundary conditions in the CFD and HAM models. In the CFD model, the surface temperature and humidity ratio as determined by the HAM model are imposed as boundary conditions in the air domain. The humidity ratio is obtained from capillary pressure and temperature using Kelvin's equation. In the HAM model, heat and moisture fluxes determined by CFD are applied as boundary conditions in the porous domain. When capillary saturation is reached at the surface of a porous material, no additional water can be taken up by the material and the excess water leads to film forming and runoff. In the present HAM model, we do not model runoff explicitly, but any water reaching the surface after capillary saturation is attained is removed by drainage and assumed not to influence other surfaces by runoff.

\section{Description of the case study}

\subsection{Geometry and air domain}

A stand-alone street canyon geometry is considered as one of the simple representations of a common urban morphology (Fig. 1a). The street canyon is composed of two identical buildings with a south-north orientation. The dimensions of the buildings are $10 \times 10 \times 50 \mathrm{~m}^{3}$. The street canyon is exposed to wind coming from west, which direction is assumed to remain constant over time. The total computational domain measures $60 \times 230 \times 250 \mathrm{~m}^{3}$. The computational grid for the air subdomain is constructed following a grid sensitivity analysis (for more details we refer to [2]). The grid consists of $1,178.080$ cells as shown in Fig. 1b. At the inlet of the computational domain, a fully developed atmospheric boundary layer profile is specified, assuming a neutral stratification, while uniform values for ambient temperature and humidity ratio are used. At the walls, standard wall functions are used while, for the ground surface, a roughness modification is considered following [11] in order to limit unwanted horizontal inhomogeneous development of the inlet velocity profile.
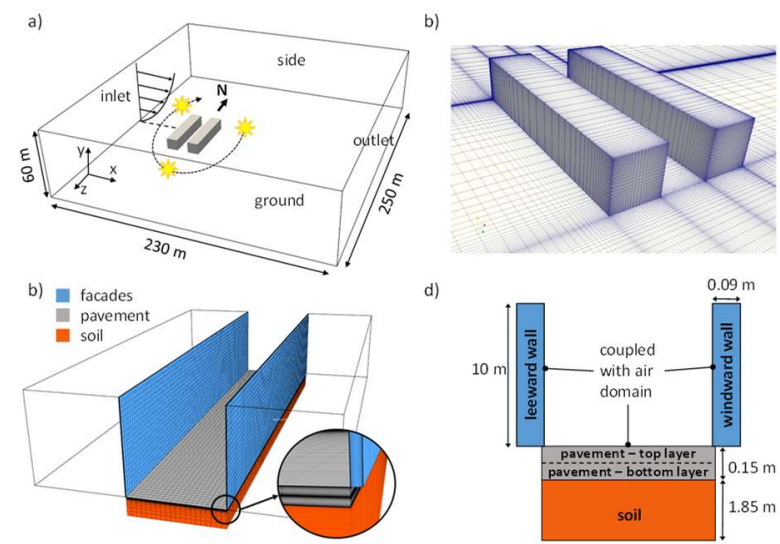

Fig. 1. a) Computational domain of street canyon with indications of wind inlet profile and of movement of the sun. b) Mesh used in the air subdomain. c) Geometry of street canyon with indication of porous media of facades, pavement and soil and grids used in these domains. d) Cross section of the porous building subdomain (figures based on [4], with permission).

\subsection{Walls and pavements}

The leeward and windward walls (Fig 1d) are finished with a $9 \mathrm{~cm}$ brick layer. The remaining part of the wall is thermally modelled by a thermal resistance of $2.5 \mathrm{~m}^{2} \mathrm{~K} / \mathrm{W}$ with a constant indoor temperature of $20^{\circ} \mathrm{C}$. At the interior surfaces of the walls no moisture transport is assumed (impermeable). The exterior surfaces of the walls have an albedo value of 0.4. All other surfaces (roofs, lateral building walls and ground outside the street canyon) are assumed to be impermeable and adiabatic. The street is composed of a pavement of $15 \mathrm{~cm}$ thickness with $1.85 \mathrm{~m}$ of soil underneath acting as heat and moisture storage. The pavement consists of two layers. The top pavement material has a thickness of $7.5 \mathrm{~cm}$, a lower porosity of $10 \%$ and pores sizes of around $10^{-5}-10^{-4} \mathrm{~m}$ (referred to as PA1). The bottom layer material has also a thickness of $7.5 \mathrm{~cm}$, but a higher porosity of $30 \%$ with coarser pores sizes of around $10^{-4}-10^{-2} \mathrm{~m}$ (PA3). It is noted that the porosity of these materials is significantly larger than those of conventional dense pavement materials, like concrete, whose porosity is typically lower showing also a low liquid permeability. In [4] we demonstrate that this double-layer pavement solution shows maximal evaporative cooling with a long lasting first drying period and high surface temperature reduction. The pavement surface has an albedo of 0.1 or 0.4 representing a dark or light coloured finishing. At $2 \mathrm{~m}$ depth, a constant temperature of $10^{\circ} \mathrm{C}$ is assumed and the surface at this 
depth is assumed to be impermeable to moisture. The computational grid of the HAM model for windward and leeward facades consists of 100,000 cells, refined towards the exterior surfaces. The grid for the street domain consists of 340,000 cells, gradually refined towards the exterior surface and the interface between different materials (Fig. 1c).

The moisture transport properties of the different materials are taken from [4]. The capillary pressure curve $w\left(p_{c}\right)$ and liquid permeability $K\left(p_{c}\right)$ for some materials are given in Fig. 2. Figure 3 gives the pore volume distribution. PA1 shows a low porosity and low capillary moisture content but high liquid permeability because of its rather coarse pores. PA3 shows a higher porosity and higher capillary moisture content because of its even coarser pores. PA3 with coarser pores shows a larger water storage capacity, while PA1 with finer pores shows a stronger capillarity. The properties of PA2 lie in between those of PA1 and PA3. In contrast, concrete shows both a low capillary moisture content and very low liquid permeability because of its very fine pore structure. For completeness, the moisture transport properties of soil are given.
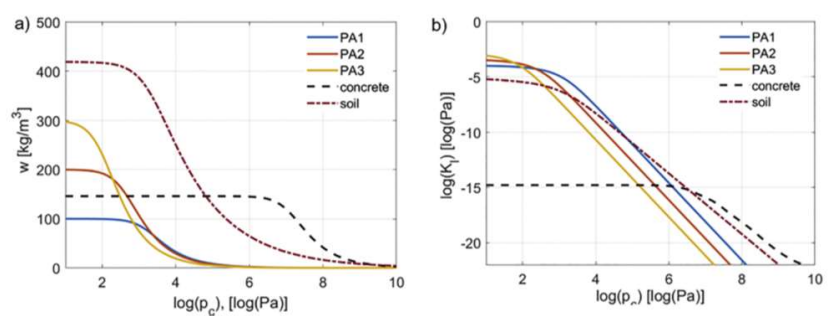

Fig. 2. a) Capillary pressure curves and b) liquid permeability curves versus capillary pressure (with permission, based on [4]).

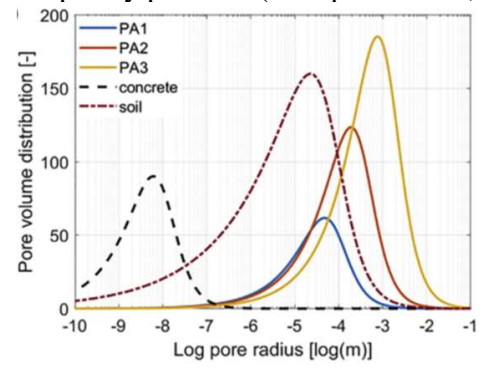

Fig. 3. Pore volume distributions (with permission, based on [4]).

\subsection{Meteorological conditions}

We consider two different meteorological conditions (MC). In MC1, the meteorological conditions are for an average sunny summer day ( $21^{\text {st }}$ of June, Zurich) with moderate air temperature and clear sky conditions [12].

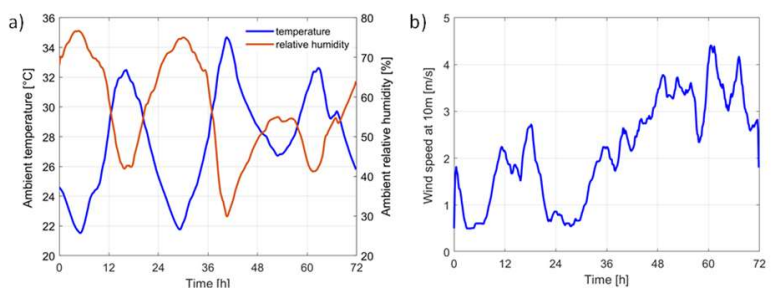

Fig. 4. a) Air temperature, relative humidity and b) wind speed at $10 \mathrm{~m}$ for three days during the heat wave in the city of Zurich of June 2017. [12].
The air temperature varies between $11^{\circ} \mathrm{C}$ and $19^{\circ} \mathrm{C}$, the $\mathrm{RH}$ between $62 \%$ and $85 \%$. In MC 2, we consider the actual meteorological conditions during the three hottest days of the heat wave of 2017 (21st, 22nd and 23 $3^{\text {rd }}$ June) in the city of Zurich (Fig. 4). The air temperature varies between $22^{\circ} \mathrm{C}$ and $35^{\circ} \mathrm{C}$, the RH between $30 \%$ and $80 \%$. The wind speed at $10 \mathrm{~m}$ is low in the first two days with values between 0.6 and $2.8 \mathrm{~m} / \mathrm{s}$. The last day is more windy with values reaching more than $4 \mathrm{~m} / \mathrm{s}$.

\section{Results}

\subsection{Meteorological conditions MC1}

In this section, we present the results for different multilayer pavement configurations (figures with permission based on [4]). In the multilayer configurations, material PA1 is combined with materials PA2 and PA3. Two configurations have PA1 on top with PA2 or PA3 in the bottom referred to as PA1-PA2 and PA1-PA3, respectively. The third configuration is with PA2 at the top and PA1 in the bottom, or PA2-PA1. As reference, the results for a single pavement layer PA1 with similar total thickness as the multilayer solutions are given. After a first dry day, the pavement is wetted during night with a wetting flux of $1 \mathrm{~mm} / \mathrm{h}$ over a period of 10 hours (blue zone in Fig. 5). The evaporative cooling effect is studied for the next two days during which drying occurs. Figs 5a$\mathrm{b}$ give the average temperature and relative humidity of the street surface over the three days. Fig. 5c gives the difference in average surface temperature between the first dry day and day 2 after the period of wetting, referred to as $\mathrm{T}_{\text {wet- }} \mathrm{T}_{\text {dry. }}$. Fig. $5 \mathrm{~d}$ gives the difference in UTCI for the same days, UTCI wet $_{\text {- UTCI }}$ ry. The differences between wet and dry days are measures for the evaporative cooling effect. We observe that, during the first dry day, the street surface reaches temperatures of more than $50^{\circ} \mathrm{C}$. The difference in surface temperature between the different pavement solutions for dry conditions is rather small. Pavements with higher porosity show slightly higher surface temperature values due to their lower thermal diffusivity meaning that less heat diffuses and is stored in the substrate.

During the second day after 10 hours of wetting by rain, drying starts and the surface temperature drops substantially due to evaporative cooling by amounts between 2 and $20^{\circ} \mathrm{C}$. The exact drop in temperature varies over time depending on the incident short wave radiation, shadowing effects, radiative exchange with walls and sky and heat transfer due to convection and evaporation. The multilayer solutions with PA1 on top (PA1-PA3, PA1PA2) show the lowest surface temperatures, indicating a higher evaporative cooling effect than for the single layer pavement PA1 and the combination PA2-PA1. The evaporative cooling effect for PA1-PA3 is highest and visible for the longest time after the wetting with temperatures $5-15^{\circ} \mathrm{C}$ lower compared to the other pavements. The reason for the higher evaporative cooling effect is that PA1 stores in its pore structure more water during wetting which, during drying, can be transported 
back to the surface by capillary forces keeping the outer layer of the pavement more and longer wet during the first drying period. The top layer PA1 from the combination PA1-PA3 stores $37 \%$ more water than in PA1-PA2 and $64 \%$ more water than in the PA1-single-layer even though the top material is the same. This is because the PA3 layer has bigger pores and thus lower suction preventing that water is sucked from the top PA1 layer, which shows smaller pores and higher suction. As such the combination PA1-PA3 results in a larger evaporative cooling effect since less water is lost towards the bottom layers, as PA3 acts as a capillary break between PA1 and soil. Water remains available to be transported back to the surface by capillary suction in the smaller pores and being evaporated at the surface leading to maximal evaporative cooling.
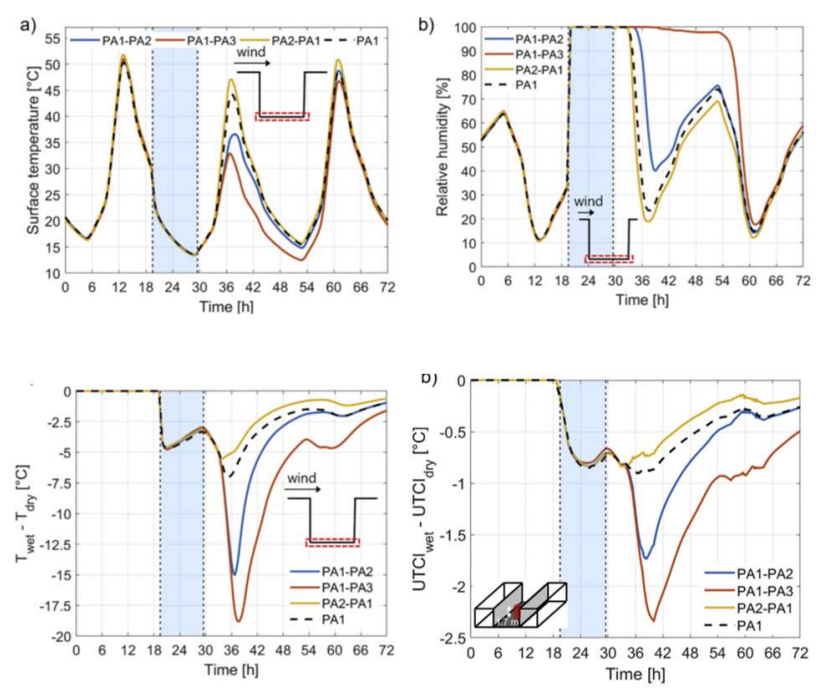

Fig. 5. Results averaged over the total street surface: (a) surface temperature; (b) relative humidity at surface; (c) drop in surface temperature comparing wet and dry days; (d) reduction in UTCI comparing wet and dry days (with permission, based on [4]).

The duration of first drying phase can be evaluated by determining the period during which the $\mathrm{RH}$ at the surface remains close to $100 \%$. It is clear that, for configurations PA1-PA3 and PA1-PA2, the duration of first drying phase is longer. In particular, the duration of the first drying phase is around five hours for PA1-PA2, while for PA1PA3 the duration is around 24 hours. Fig. 5d gives the difference in UTCI between wet and dry days for a person standing in the middle of the street canyon, where wind velocity, temperature and relative humidity are evaluated at a pedestrian height of $1.7 \mathrm{~m}$. The UTCI can drop up to $2.5^{\circ} \mathrm{C}$ for the combination PA1-PA3, showing that welldesigned permeable pavements can be efficiently used to reduce the air and surface temperatures leading to more comfortable conditions.

\subsection{Meteorological conditions MC2}

The analysis in the first case study MC1 with normal summer conditions and a long rain event showed the potential of evaporative cooling of the pavement combination PA1-PA3. In the case study MC2, we analyse the effect of evaporative cooling by artificial wetting during an extreme heat event of three days. The questions we want to answer are 'how much', 'when' and 'how long' do we have to artificially wet the pavement to achieve a maximal cooling effect by evaporation. The case study is a 3D street canyon. However, 3D simulations, especially the CFD model, require quite long calculation times to adequately answer these questions. Therefore, we first perform 1D simulations for the pavement with soil and determine the optimal wetting process to achieve the maximal reduction in surface temperature and longest duration of the first drying period. To obtain as accurate results as possible in 1D, these simulations are performed with environmental conditions obtained from a full 3D dry simulation: i.e. the air temperature and $\mathrm{RH}$, heat transfer coefficient, and net shortwave radiation. For simplicity, we account only for longwave radiation exchange with the sky, disregarding in this 1D study longwave radiation exchange with street canyon walls. Comparing 1D and 3D simulation results for one case of artificial wetting (see later) showed that the $1 \mathrm{D}$ simulation results are sufficiently accurate to perform a first optimisation of the artificial wetting process (although an underestimation in surface temperature in $1 \mathrm{D}$ is observed due to the simplification of neglecting the radiative exchange with the walls).

Fig. 6 shows the temperature and $\mathrm{RH}$ at the surface for artificial wetting for different values of wetting flux of $1,2,2.5$ and $3 \mathrm{~mm} / \mathrm{h}$ for a total wetting period of 2 hours. The wetting starts at 8:00 in the morning. We found that a wetting flux of $2.5-3 \mathrm{~mm} / \mathrm{h}$ shows sufficient evaporative cooling during 24 hours with a maximal drop of more than $20^{\circ} \mathrm{C}$ in surface temperature. This means that a total amount of $5 \mathrm{~mm} /$ day is sufficient for having a maximal evaporative cooling effect during that day. In Fig. 7, we repeated for two consecutive days a wetting with $5 \mathrm{~mm}$ /day during 2 hours at a flux of $2.5 \mathrm{~mm} / \mathrm{h}$ starting at 8:00 am. The results show that the same evaporative cooling effect is achieved by repeating this wetting scenario every day during the heat wave.

We now move to the question 'how long' artificial wetting is needed. We shorten the duration of wetting while keeping the total amount of wetting constant (increasing the wetting flux), keeping in mind that too high wetting fluxes should be avoided since for these fluxes the material surface can attain capillary saturation leading to runoff. Fig. 8 shows the temperature and RH at the surface for artificial wetting with a total amount of 5 $\mathrm{mm} /$ day for different wetting periods of $10 \mathrm{~min}, 30 \mathrm{~min}$, 1 and 2 hours. 

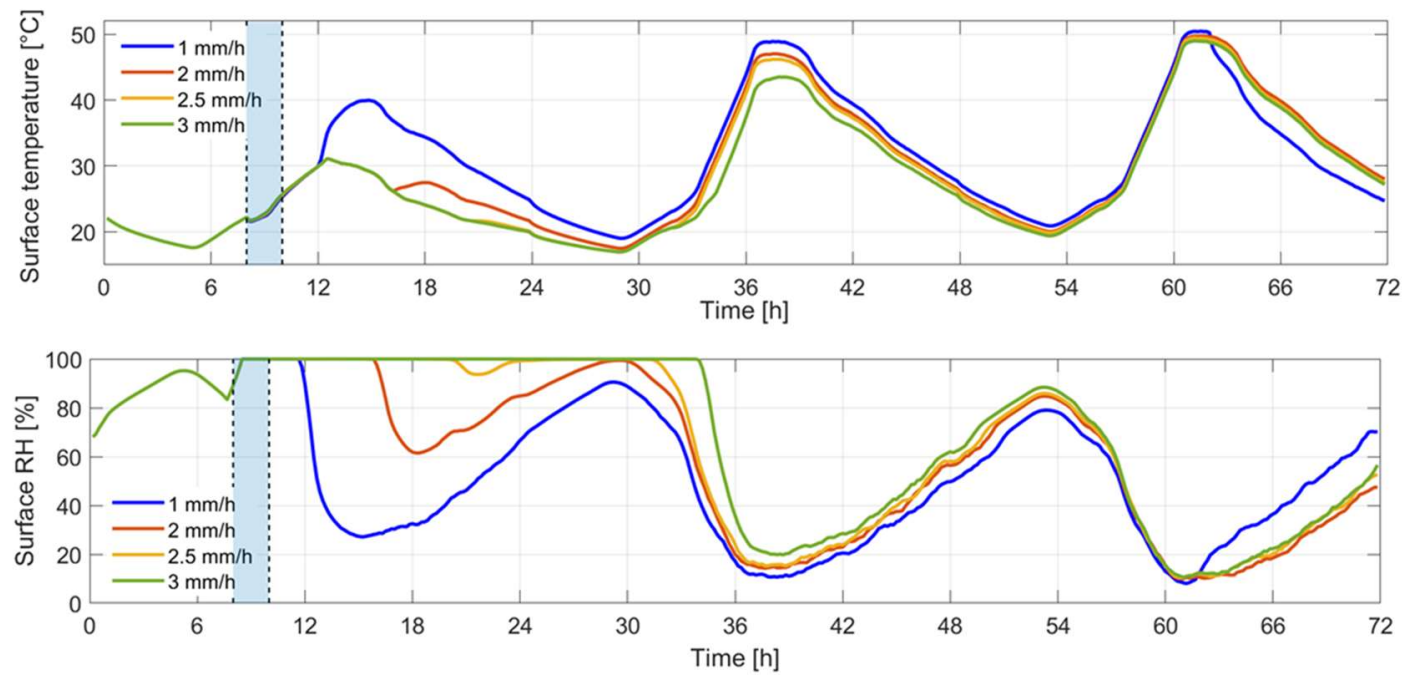

Fig. 6. 1D results of surface temperature (top) and relative humidity RH (bottom) for three consecutive days during a heat wave period. Effect of wetting flux over a wetting period of 2 hours.
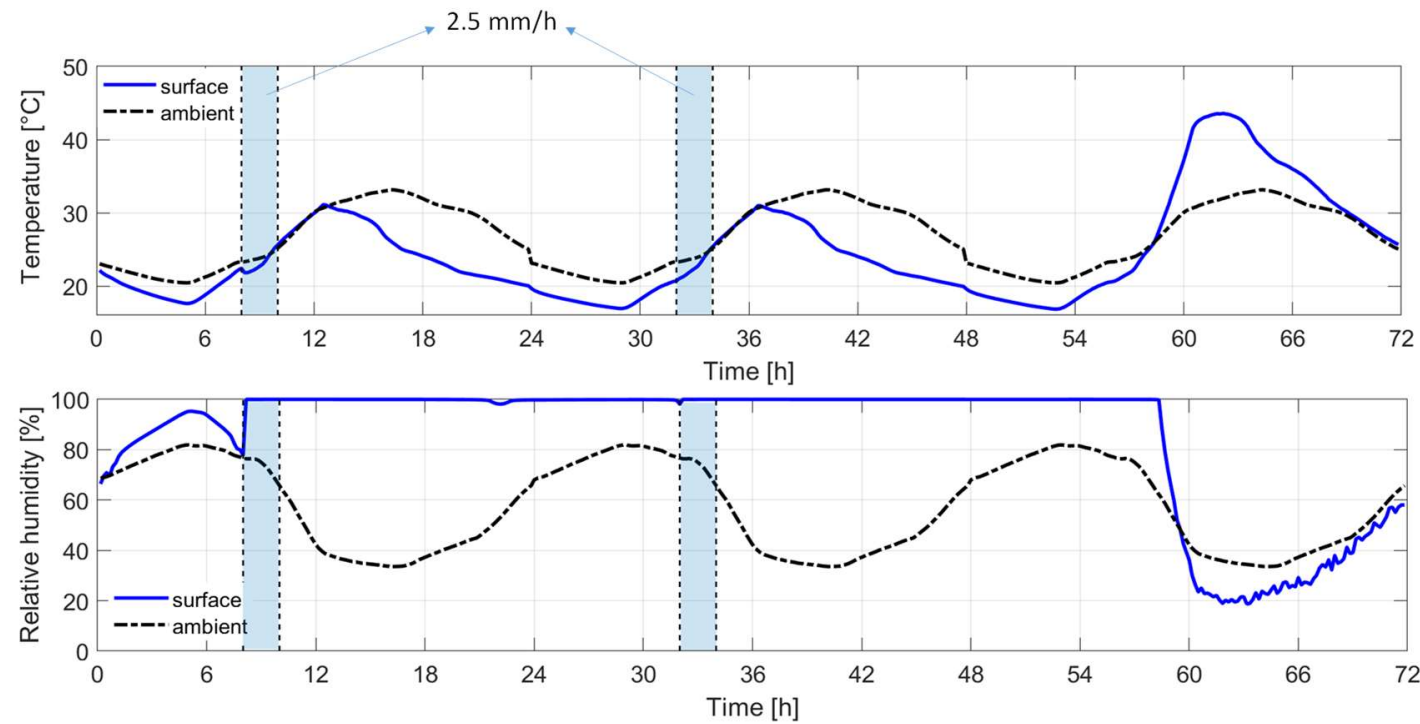

Fig. 7. 1D results of surface temperature and relative humidity RH for three consecutive days during a heat wave period. Two days of wetting with $5 \mathrm{~mm} /$ day during two hours starting at $8 \mathrm{am}$.
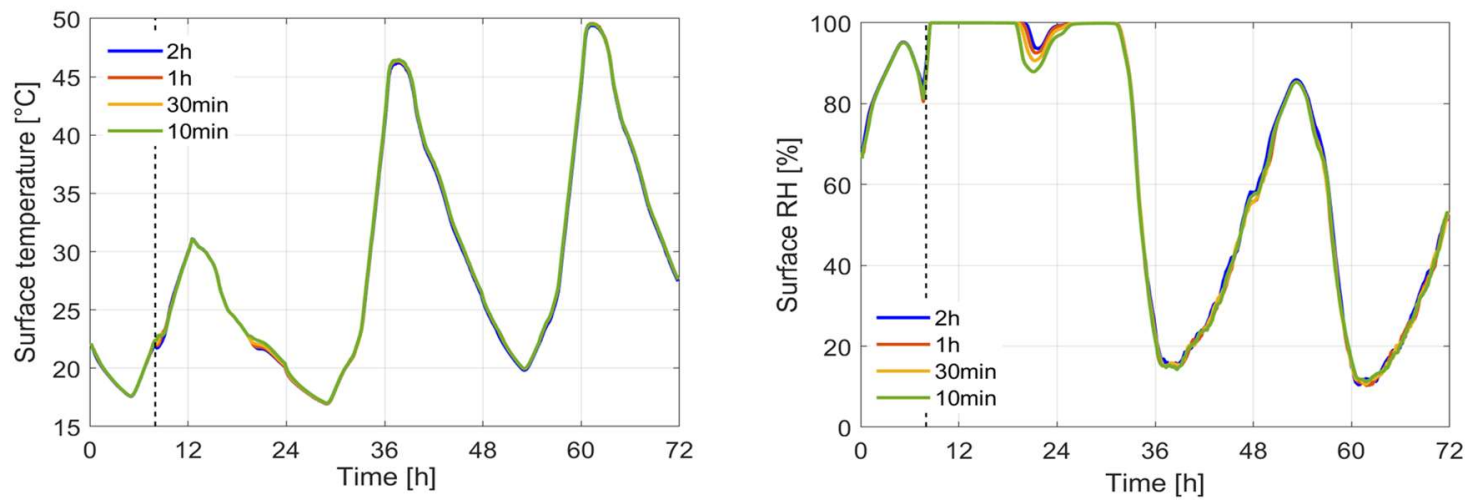

Fig. 8. 1D results of surface temperature and relative humidity RH for three consecutive days during a heat wave period. Effect of duration of wetting period for a total amount of wetting of $5 \mathrm{~mm} /$ day. 

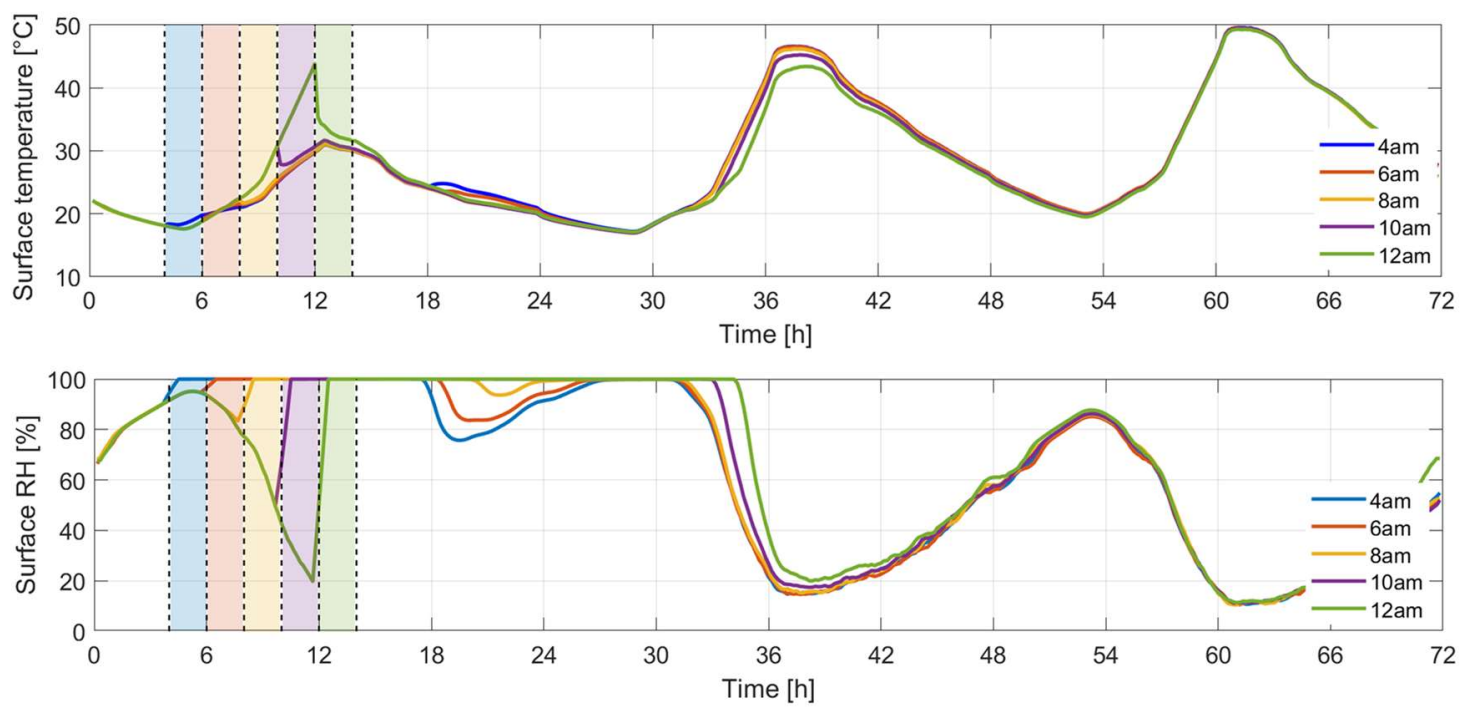

Fig. 9. 1D results of surface temperature and relative humidity RH for three consecutive days during a heat wave period. Effect of starting time of wetting for a total amount of wetting of $5 \mathrm{~mm} /$ day.
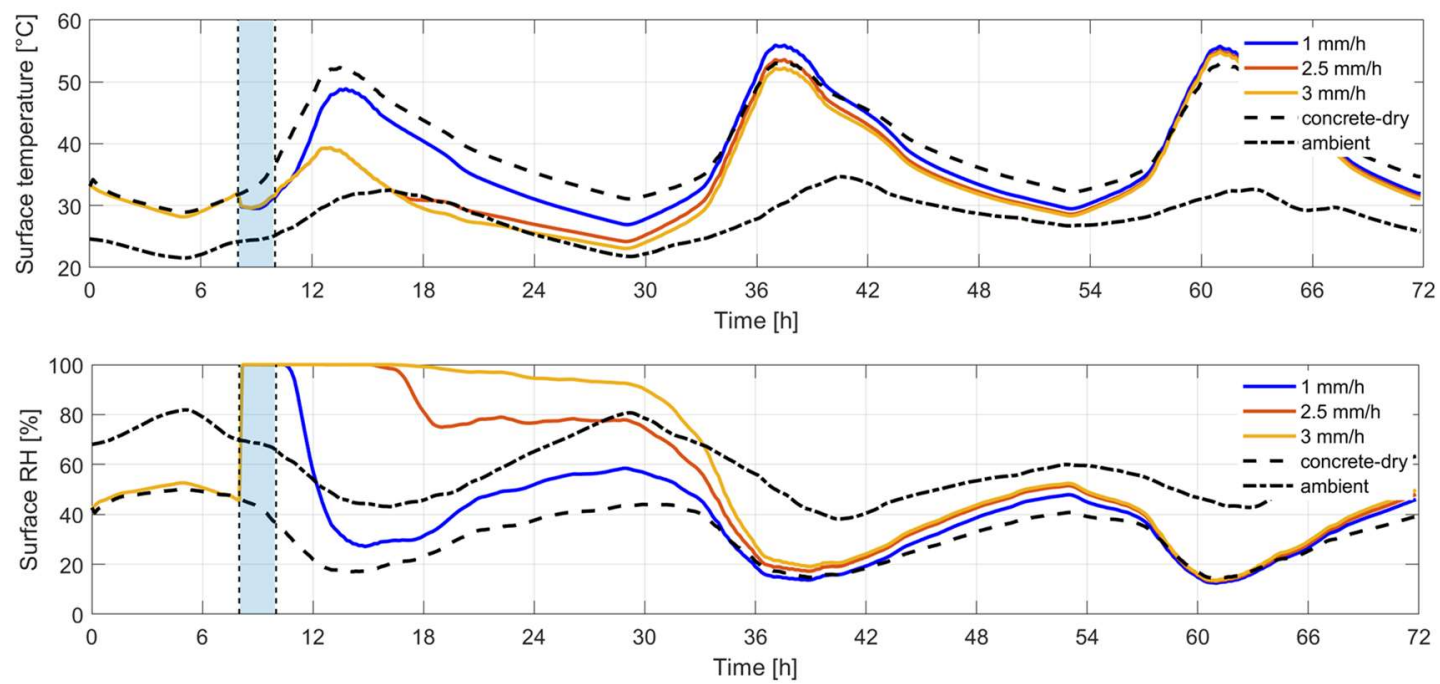

Fig. 10. 3D results of surface temperature and relative humidity $\mathrm{RH}$ for three consecutive days during a heat wave period. Effect of wetting flux for a total amount of wetting of $5 \mathrm{~mm} /$ day starting at 8:00 and wetting period of 2 hours.

We observe almost no difference in surface temperature and duration of first drying period for all wetting periods, showing that a short wetting duration of 10 minutes is the most feasible wetting duration. During these large wetting fluxes, capillary saturation was never reached at the surface, mainly due to the high permeability of the top layer pavement PA1. We finally move to the question 'when' artificial wetting has to start. Fig. 9 shows the surface temperature and $\mathrm{RH}$ at the surface for artificial wetting with a total amount of $5 \mathrm{~mm} /$ day with a wetting period of 2 hours starting at 4:00, 6:00, 8:00, 10:00 and 12:00 am. We observe that, when wetting occurs between 8:00 and 10:00 am, we achieve the highest evaporative cooling effect during the day and night cycle. Earlier wetting leads to less evaporative cooling effect during the evening, and wetting later than 10:00 am lets the air temperature increase early in the morning when the street is sunlit. In general, we can state that wetting should occur preferentially before the sun starts to irradiate the street too much to have a maximal cooling effect.
We finally perform a 3D simulation in order to confirm the results obtained in the 1D simulations. Fig. 10 shows the temperature and $\mathrm{RH}$ at the surface for artificial wetting with wetting fluxes of $1,2.5$.and $3 \mathrm{~mm} / \mathrm{h}$ and a wetting period of 2 hours starting at 8:00 am. The figure also shows the ambient temperature and the results for dry concrete for comparison. The simulations confirm that such wetting conditions lead to a maximal evaporative cooling during the same day and night. The $1 \mathrm{D}$ simulation predicts maximal surface temperatures around $50^{\circ} \mathrm{C}$ in dry conditions, a reduction in surface temperature by $20^{\circ} \mathrm{C}$ and a duration of first drying period of 24 hours. In $3 \mathrm{D}$, the maximal temperature is around $55^{\circ} \mathrm{C}$, with the same maximal evaporative cooling effect of $20^{\circ} \mathrm{C}$ and a duration of first drying period of around 20 hours. This shows that 1D simulations can be used to guide the optimisation process of 'smart wetting' although an offset of $5^{\circ} \mathrm{C}$ is observed in maximal surface temperature, mainly due an overestimation of cooling to the sky by longwave radiation in the $1 \mathrm{D}$ simulation. 
We finally note that further 3D simulations are still running and systematic results on 'smart wetting' including also the effect on pedestrian urban comfort will be reported at the conference.

\section{Conclusions}

A microclimate model is used to design the optimal artificial wetting strategy for multilayer street pavements in order to guarantee a maximal evaporative cooling effect as mitigation measure for local heat islands in street canyons during heat waves. The microclimate model is composed of a computational fluid dynamics (CFD) model implemented in OpenFOAM solving for turbulent heat, air and moisture transport in the air domain, and coupled to models for heat and moisture transport in urban porous materials, solar shortwave and longwave transport and wind driven rain. Studying the evaporative cooling effect of a rain event during summer days on the local microclimate in a street canyon, we found that the proper selection of a multilayer pavement system can allow a maximal cooling effect with a first drying period lasting for a full day and night cycle. Essential for the multilayer pavement solution is that the top layer allows for a fast and sufficient storage of water during the wetting period, while during drying guaranteeing suction of the liquid water back to the top surface through sufficiently small pores, hence enabling maximal evaporative cooling during first drying period. The second layer with bigger pores guarantees that during the wetting period only a limited amount of water is lost from at the bottom of the top pavement layer due to capillary break effect. Having defined an optimal multilayer pavement system, the optimal wetting protocol, referred to as 'smart wetting', is determined in terms of 'how much', 'when' and 'how long' the artificial wetting of the pavement has to last. It is shown that a $1 \mathrm{D}$ simulation of the heat and moisture transport in a pavement and underlying soil with proper boundary conditions can serve as a simplified method to allow finding the optimal solution. We found that a daily amount of $5 \mathrm{~mm}$ of artificial wetting over 10 minutes in the morning preferentially between 8:00 and 10:00 am guarantees a maximal evaporative cooling for a day and night cycle.

This research project is supported by the Swiss Competence Center for Energy Research SCCER FEEB\&D of the Swiss Innovation Agency lnnosuisse and the Swiss National Science Foundation (SNF) - Project no. 169323, CTI.1155002539.

\section{References}

1. P. Moonen, T. Defraeye, V. Dorer, B. Blocken, \& J. Carmeliet, Urban physics: effect of the micro-climate on comfort, health and energy demand. Front. Arch. Res. 10 (3), 197-228 (2012)

2. A. Kubilay, D. Derome, and J. Carmeliet. Coupling of physical phenomena in urban microclimate: A model integrating air flow, wind-driven rain, radiation and transport in building materials. Urban Climate, 24, 398-418 (2018).
3. A. Kubilay, D. Derome, and J. Carmeliet. Impact of evaporative cooling due to wetting of urban materials on local thermal comfort in a street canyon. Sustainable Cities and Society, 49, 101574, (2019).

4. A. Ferrari, A. Kubilay, and J. Carmeliet. The use of permeable and reflective pavements as a potential strategy for urban heat island mitigation. Urban Climate, 31, 100534 (2020)

5. D. Fiala, G. Havenith, P. Bröde, B. Kampmann, G. Jendritzky, G., 2012. UTCI-Fiala multi-node model of human heat transfer and temperature regulation. Int. J. Biometeorol. 560 (3), 429-441. (2012).

6. P. Maillard, F. David, M. Dechesne, J.-B. Bailly, and E. Lesueur. Characterization of the urban heat island and evaluation of a road humidification mitigation solution in the district of la Part-Dieu, Lyon (france). Tech. Sci. Methodes, 6, 23-35 (2014).

7. M. Bouvier, A. Brunner, and F. Aimé. Nighttime watering streets and induced effects on the surrounding refreshment in case of hot weather. The city of Paris experimentations. Tech. Sci. Méthodes, 12, 43-55 (2013).

8. M. Hendel, M. Colombert, Y. Diab, and L. Royon. An analysis of pavement heat flux to optimize the water efficiency of a pavement-watering method. Applied thermal engineering, 78, 658-669 (2015).

9. A. Kubilay, D. Derome, B. Blocken, J. Carmeliet, CFD simulation and validation of wind-driven rain on a building facade with an eulerian multiphase model. Build. Environ. 61, 69-81 (2013)

10. OpenFOAM, 2015. OpenFOAM v2.4.0 User Guide. OpenCFD Ltd.

11. B. Blocken, T. Stathopoulos, and J. Carmeliet. CFD simulation of the atmospheric boundary layer: wall function problems. Atmospheric environment, 41(2), 238 - 252 (2007).

12. Meteonorm, 2000. Global Meteorological Database for Solar Energy and Applied Climatology. Meteotest, Bern, Switzerland. 